Short Technical Report

\title{
Streamlined Approach to Functional Analysis of Promoter-Region Polymorphisms
}

BioTechniques 33:412-418 (August 2002)

\author{
S.L. Coleman, B. Hoogen- \\ doorn, C. Guy, S.K. Smith, \\ M.C. O'Donovan, and P.R. \\ Buckland \\ University of Wales College of \\ Medicine, Cardiff, UK
}

\section{INTRODUCTION}

Data suggesting that the human genome contains fewer genes than most researchers have previously predicted underscore the potential importance of variation in gene regulation in determining disease susceptibility and other inherited phenotypic variables (7). Because only a small proportion of polymorphisms will alter gene expression, improved methods for identifying such functional variants are crucial to finding the inherited basis for illnesses, primarily those of a complex nature.

Most genes have many cis-acting regulatory regions, and polymorphisms occurring in any of these regions could potentially affect gene expression. How ever, the most precisely defined are promoters, and to date research on the genetic basis for variation in gene expression has focused on this region. Unfortunately, typical protocols for screening promoters, identifying polymorphisms, and subsequent functional analysis $(1,2)$ are fairly laborious; consequently, relatively few promoters have been systematically screened for even common variants that alter gene expression. Here we present the development of reagents and an optimized protocol that allows two researchers to prepare at least 20 clones (10 allelic pairs) and test them for effects on gene expression in each of three cell lines per week.

\section{MATERIALS AND METHODS}

\section{Promoter Selection and Polymorphism Identification}

DNA promoter sequences from the Eukaryotic Promoter Database (EPD; www.epd.isb-sib.ch) (8) were amplified by PCR using QiaTaq ${ }^{\circledR}$ (Qiagen, Valencia, CA, USA) to include approximately 500 bases $5^{\prime}$ to the start of transcription of the selected gene. In selecting the promoter sequence, the specifications for the $3^{\prime}$ primer were, in order of priority, that $(i)$ the start of transcription was included within the amplimer, (ii) it did not amplify an ATG within the 5'-untranslated region (UTR), and (iii) it was within 50 bp $3^{\prime}$ to the start of transcription to minimize the effects of the $5^{\prime}$ UTR. Approximately $5 \mu \mathrm{L}$ crude PCR product from each of 16 individuals' DNA were screened for polymorphisms using denaturing HPLC under conditions selected by DHPLCMelt (http:// insertion.stanford.edu/melt.html) as described (5). Fragments yielding chromatograms indicating heteroduplex formation were sequenced using BigDye ${ }^{\mathrm{TM}}$ terminator chemistry (Applied Biosystems, Foster City, CA, USA) to confirm and characterize polymorphisms.

\section{Cloning}

For high throughput, we required a cell lines (HEK293t, TE671, and JEG3)

with similar resources. 
strategy of direct cloning into the luciferase expression vector pGL3 (Promega, Madison, WI, USA). To create a high-efficiency pGL3 T/A cloning vector, we first inserted an EcoRV site using standard procedures into the pGL3-basic multiple cloning site to create pGL3-basic-RV. This was cut with EcoRV $(10 \mu \mathrm{g}$ plasmid, $120 \mathrm{U}$ EcoRV, $100 \mu \mathrm{g} / \mu \mathrm{L}, \mathrm{BSA}, 10 \mu \mathrm{L}$ NEB buffer 3 in $100 \mu \mathrm{L}$ ) at $37^{\circ} \mathrm{C}$ for $18 \mathrm{~h}$ and purified using a Qiagen plasmid miniprep kit. A $3^{\prime} \mathrm{T}$ overhang was added by incubating $10 \mu \mathrm{g}$ cut vector at $75^{\circ} \mathrm{C}$ for $3 \mathrm{~h}$ in $10 \times$ PCR buffer (Qiagen), $20 \mu \mathrm{L}$ $25 \mathrm{mM} \mathrm{MgCl}_{2}, 8 \mu \mathrm{L} 100 \mathrm{mM}$ dTTP, 2 $\mu \mathrm{L}$ Taq DNA polymerase (Qiagen) 10 $\mathrm{U}$ in $200 \mu \mathrm{L}$ final volume (6). Unpurified product was stored in single-use aliquots at $-80^{\circ} \mathrm{C}$.

DNA from heterozygous individuals was amplified using Expand ${ }^{\mathrm{TM}} \mathrm{High}$ Fidelity DNA polymerase (Roche Applied Science, Indianapolis, IN, USA) to minimize misincorporation of nucleotides. PCR products to be cloned were purified using a Qiagen spin column. Amplimer (15 ng) and $50 \mathrm{ng}$ prepared T-vector were ligated in $10 \mu \mathrm{L}$ using T4 DNA ligase (Promega) at $4{ }^{\circ} \mathrm{C}$ overnight or at room temperature for 1 h. After ligation, $1 \mu \mathrm{L}$ of the mixture was incubated at $37^{\circ} \mathrm{C}$ for $2 \mathrm{~h}$ with $20 \mathrm{U}$ EcoRV to cut any residual plasmid that lacked the additional $3^{\prime} \mathrm{T}$ and had thus recircularized (this procedure was not carried out where the insert contained an EcoRV site). Approximately onetenth of the recut ligation mixture (i.e., approximately $500 \mathrm{pg}$ equivalent of vector) was used to transform $100 \mu \mathrm{L}$ SURE ${ }^{\circledR} 2$ supercompetent cells (Stratagene, La Jolla, CA, USA) (3). Colonies were picked using a sterile pipet tip, suspended in $25 \mu \mathrm{L}$ water, and heated to $97^{\circ} \mathrm{C}$ for $5 \mathrm{~min}$. The suspension (1.5 $\mu \mathrm{L}$ ) was analyzed by PCR using the vector primers to measure the efficiency of the cloning (GL2 and RV3; Promega; annealing temperature, $56^{\circ} \mathrm{C}$ ). Correct orientation was determined using one gene-specific primer and one plasmidspecific primer (RV3; Promega; annealing temperature, $58^{\circ} \mathrm{C}$ for all inserts). PCRs carried out with a variety of com binations of insert-specific and plasmid primers showed that approximately $0.4 \%$ of the colonies had been transformed with more than one plasmid.

\section{Identification of Allelic Pairs}

At least six clones, with the insert correctly orientated, were picked to identify allele pairs. To limit the num ber of clones to be sequenced, allele pairs were identified by mixing the PCR products from pairs of clones and looking for heteroduplex formation by denaturing HPLC under the same conditions originally required for polymorphism detection. Each mixture was heated to $97^{\circ} \mathrm{C}$ for $3 \mathrm{~min}$ and allowed to re-anneal before analysis by denaturing HPLC. The component plasmids of a mixture showing the signature of a heterozygote were isolated from the SURE 2 cells using standard procedures, sequenced to confirm fidelity, and used to transfect eukaryotic cells in culture. The use of at least six clones gives a $98 \%$ chance of isolating both allele pairs, assuming equal allelic representation in clones. For 49 promoters where six colonies were screened, 48 yielded both alleles in accordance with predicted results.

\section{Cell Culture}

The ability of each sequence to promote transcription of the luciferase gene was tested transiently in human cell lines HEK293t (human embryo kidney, a gift from GlaxoSmithKline), TE671 [human medulloblastoma; identical to rhabdomyosarcoma RD cell line, European Collection of Cell Cultures (ECACC) no. 85111502], and JEG-3 (human placenta). The latter two lines were obtained from the ECACC. Cell lines were transfected with plasmid using LIPOFECTAMINE ${ }^{\mathrm{TM}}$, follow ing the manufacturer's protocol (Invitrogen, Carlsbad, CA, USA) with modifications described below. Cell lines were cultured according to ECACC specifications at $37^{\circ} \mathrm{C}$ with $5 \%$ $\mathrm{CO}_{2}$. Cells were seeded into black, clear-bottomed, 96-well, luminometric plates (Applied Biosystems) at approximately $60 \%$ confluence the day before transfection. Plates seeded with HEK293t were coated with lysine (Sigma, St. Louis, MO, USA) before seeding. Before transfection, all plasmids were quantitated fluorimetrically using PicoGreen ${ }^{\circledR}$ (Molecular Probes, Eugene, OR, USA) and a TD-700 fluo- rimeter (Turner Designs, Sunnyvale, CA, USA). HEK293t cells were transfected with $40 \mathrm{ng} /$ well DNA, $1 \mu \mathrm{L}$ LIPOFECTAMINE, TE671 were transfected with $80 \mathrm{ng} /$ well DNA, 0.25 $\mu \mathrm{L} /$ well LIPOFECTAMINE, $0.75 \mu \mathrm{L} /$ well PLUS-reagent (Invitrogen), and JEG3 were transfected with $80 \mathrm{ng} /$ well DNA, $0.5 \mu \mathrm{L} /$ well LIPOFECTAMINE, $0.5 \mu \mathrm{L} /$ well PLUS-reagent. To provide a control for transfection efficiency, cells were co-transfected with a secreted placental alkaline phosphatase reporter gene plasmid driven by the cytomegalovirus promoter (CMV-SPAP; a gift from GlaxoSmithKline). A ratio of approximately 1:4000 control to test DNA was used (HEK293t and TE671 at $0.01 \mathrm{ng} /$ well and JEG3 with CMVSPAP 0.02 at ng/well). Cell lines were transfected overnight in serum-free medium, which was replaced with complete heat-inactivated medium (PAA Laboratories, Yeovil, UK) and incubated for a further $24 \mathrm{~h}$.

SPAP activity was measured in the culture medium after transferring to a second 96-well plate using a phosphalight $^{\mathrm{TM}}$ kit (Tropix, Bedford, MA, USA) according to manufacturer's instructions. Luciferase activity in the remaining cells was measured in the original plate using a LucScreen ${ }^{\circledR}$ assaying kit (Tropix), which was chosen because of an extended luciferase half-life of 4-5 h (9). Both plates were read on a single photon counting luminometer (TR717; Berthold Technologies, Redbourn, UK) for 1-10 s/well.

\section{RESULTS AND DISCUSSION}

\section{Cloning Allelic Pairs}

Identifying homozygous individuals for each allele requires genotyping before cloning and is impractical for rare alleles. Therefore, for efficiency, we elected to routinely clone the PCR products from a heterozygote. Thirtytwo colonies were picked for each promoter. The average percentage of colonies with a full-length insert was $68 \%$ $(n=150)$, while the average number of inserts in the correct orientation was approximately half this percentage, as predicted by chance. Approximately $50 \%$ of the clones without full-length 


\section{High-Throughput M ethods}

inserts gave a PCR product with the RV3 and GL2 primers of variable length, either larger or smaller than the expected 198 bp of the uncut plasmid, pGL3-basic-RV, although this was highly variable between experiments. Sequencing a selection of these clones showed that the plasmids resulted from partial deletions following the ligation of the insert and were not due to the presence of an uncut or recircularized native plasmid.

A number of procedures were attempted to further increase the efficiency of T/A cloning. These included agarose gel purification of the vector after EcoRV digestion before the addition of Ts and also after re-ligation of the vector after $3^{\prime} \mathrm{T}$ addition to remove plasmid lacking $3^{\prime}$ Ts. We also designed primers to contain a $5^{\prime} \mathrm{G}$, as it has been shown that this base enhances the extra A added to the 3'-end of PCR products by Taq DNA polymerase (4).

Table 1. Optimization of DNA:Transfection Reagent for HEK293t Cells

\begin{tabular}{|ccccccccc}
\hline HEK293t & \multicolumn{7}{c|}{$\mu$ DNA/Well } \\
\hline $\begin{array}{c}\text { LIPOFECTAMINE } \\
(\mu \mathrm{L})\end{array}$ & 0.42 & 0.28 & 0.21 & 0.14 & 0.1 & 0.07 & 0.04 & 0.02 \\
\cline { 2 - 9 } 2 & 0.4 & 0.8 & $\mathbf{1 2 . 4}$ & $\mathbf{3 0 . 4}$ & $\mathbf{2 6 . 9}$ & $\mathbf{1 7 . 9}$ & 6.3 & 0.9 \\
1.75 & 0.7 & 1.8 & 3.0 & $\mathbf{3 8 . 7}$ & $\mathbf{5 0 . 1}$ & $\mathbf{4 5 . 1}$ & 8.1 & 1.3 \\
1.5 & 2.2 & 1.3 & 0.7 & $\mathbf{2 8 . 7}$ & $\mathbf{2 7 . 4}$ & $\mathbf{6 6 . 8}$ & $\mathbf{1 3 . 4}$ & 2.6 \\
1.25 & 2.7 & 0.4 & 0.6 & 6.3 & $\mathbf{2 3 . 9}$ & $\mathbf{1 0 . 1}$ & $\mathbf{2 2 . 2}$ & 4.4 \\
1 & 1.2 & 0.7 & 0.5 & 3.2 & 9.3 & $\mathbf{3 4 . 2}$ & $\mathbf{2 6 . 1}$ & $\mathbf{1 2 . 9}$ \\
0.75 & 4.0 & 1.6 & 1.4 & 0.7 & 0.7 & 0.5 & $\mathbf{2 2 . 1}$ & $\mathbf{3 4 . 5}$ \\
0.5 & 3.2 & 2.3 & 3.7 & 5.0 & 2.2 & 0.5 & 0.9 & $\mathbf{3 5 . 8}$ \\
0.25 & 0 & 0 & 0 & 0.1 & 0.1 & 1.2 & 1.2 & 0.2
\end{tabular}

The figures are $\mathrm{cps} \times 10^{-3}$ luciferase. Values above $10000 \mathrm{cps}$ are highlighted.

None of these procedures reduced the number of colonies lacking a fulllength insert (data not shown).

Approximately $10 \%$ of the sequenced clones had a spurious base change caused by a PCR error. However, this exaggerates the true error rate, since only pairs of plasmids giving the pattern of a heterozygous mixture on denaturing HPLC were selected for 
sequencing. Spurious polymorphisms were exclusively substitutions, with the following exceptions: $(i)$ loss of a $\mathrm{T}$ from either of the cloning sites such that the expected sequence of GATTNN was replaced with GATNN; (ii) low-fidelity copying of poly(A) or poly(T) stretches, or dinucleotide repeat sequences such as CACA with respect to the length of the sequence (note that the apparent polymorphism of such sequences is frequently seen in the genomic sequencing, but the presence of five or six different "alleles" from one individual confirms the presence of sequence error); and (iii) spurious polymorphism in the plasmid. The latter was rare, but mutation of the luciferase coding sequence has been known to inactivate the protein.

We empirically determined the optimum concentration of DNA and the ratio of DNA to transfection reagent for each cell line. The relationship between concentration of plasmid and the ratio of DNA to LIPOFECTAMINE was calculated for each cell line using the pGL3promoter vector. Table 1 shows the data for the HEK293t cells. It can be seen that the ratio of DNA to LIPOFECTAMINE was the most critical variable, the absolute amount of DNA being less so. As a compromise between cost and a desire to avoid pipetting small volumes of LIPOFECTAMINE (which would result in a tendency to greater sample variation because of manipulation error), subsequent experiments were carried out using $1 \mu \mathrm{L}$ LIPOFECTAMINE and 40 ng DNA.

Optimum ratios of test to control plasmid were identified for each cell line to provide a good signal for the SPAP assay without interfering significantly with the expression of luciferase due to promoter competition. At ratios higher than 1:4000 CMV-SPAP to luciferase plasmid, the luciferase activity declined.

The HEK-293t cell line is a derivative of the HEK-293 line, which allows high-copy replication plasmids containing the simian virus 40 (SV40) origin. Of note is that the pGL3-promoter plasmid contains the SV40 promoter and displays low activity in many other cells. It is thus a poor positive control for transfection in JEG-3, TE671, and some other cell lines. Similarly, an SV40-SPAP plasmid is not suitable for an internal control.
In a representative assay, the $\mathrm{CV}$ for 10 data sets averaged 0.38 for luciferase measurements alone and 0.20 when corrected with SPAP, which controls for such factors as variation in cell number, transfection efficiency, pipetting errors, etc. Although we have been unable to complete a systematic review of the literature, this figure is comparable with the more labor-intensive methods (e.g., References 1 and 2). The intra-assay reproducibility was tested by comparing two plasmid preparations, each of 17 individual alleles in the same assay. The Pearson correlation between the pairs was $0.988(P<0.0005)$. To measure inter-assay variability, three separate plasmid preparations of an allele pair were analyzed in three individual assays. In the three assays, allele B had promoter activity, relative to allele $\mathrm{A}$, of $66 \%$, $61 \%$, and $67 \%$, respectively $(\mathrm{CV}=0.05)$. However, the absolute activity of any allele varies between assays by up to several-fold, and comparisons between any two or more alleles must be done in the same assay.

In summary, we have developed an integrated, streamlined protocol, based on procedures that have been described before for small-scale work, that permits fairly rapid identification, cloning, and analysis of promoter polymorphisms in a functional assay system. To increase throughput, we adopted a 96-well plate approach. The assay conditions we describe routinely offer a higher rate of analysis than traditional protocols without compromising reproducibility.

\section{ACKNOWLEDGMENTS}

This work was funded by the Medical Research Council, UK.

\section{REFERENCES}

\footnotetext{
1.Brinkman, B.M.N., D. Zuijdgeest, E.L. Kaijzel, F.C. Breedveld, and C.L. Verweij. 1996. Relevance of the tumour necrosis factor alpha (TNF $\alpha)-308$ promoter polymorphism in TNF $\alpha$ gene regulation. J. Inflammation 46:31-41.

2.Edenberg, H.J., R.E. Jerome, and M. Li. 1999. Polymorphism of the human alcohol dehydrogenase 4 (ADH4) promoter affects gene expression. Pharmacogenetics 9:25-30.

3.Greener, A. 1990. E. coli SURE Strain: clone "unclonable" DNA. Strategies 3:5-6.
}

4.Hu, G. 1993. DNA polymerase-catalysed addition of non-templated extra nucleotides to the 3' end of a DNA fragment. DNA Cell Biol. 12:763-770

5.Jones, A.C., J. Austin, N. Hansen, B. Hoogendoorn, P.J. Oefner, J.P. Cheadle, and M.C. O'Donovan. 1999. Optimal tem perature selection for mutation detection by denaturing HPLC and comparison to singlestranded conformation polymorphism and heteroduplex analysis. Clin. Chem. 45:11331140.

6.Marchuk, D., M. Drumm, A. Saulino, and F.S. Collins. 1991. Construction of T-vectors, a rapid and general system for direct cloning of unmodified PCR products. Nucleic Acids Res. 19:1154.

7.Peltonen, L. and V.A. McKusick. 2001. Genomics and medicine. Dissecting human disease in the postgenomic era. Science 291:1224-1229.

8.Praz, V., R.C. Périer, C. Bonnard, and P. Bucher. 2002. The Eukaryotic Promoter Database, EPD: new entry types and links to gene expression data. Nucleic Acids Res. 30:322-324.

9.Roelant, C.H., D.A. Burns, and W. Scheirer. 1996. Accelerating the pace of luciferase reporter gene assays. BioTechniques 20:914917.

Received 8 March 2002; accepted 17 May 2002.

Address correspondence to:

Dr. Paul Buckland

Department of Psychological Medicine

University of Wales College of Medicine Heath Park, Cardiff CF14 4XN, UK

e-mail: buckland@cf.ac.uk

For reprints of this or any other article, contact Reprints@BioTechniques.com 